\title{
A Spectral Power based Index using Wavelet Analysis of EEG for Drowsiness Detection
}

\author{
Yashwanth Vyza \\ École Polytechnique Fédérale de Lausanne \\ Switzerland
}

\author{
Suman Dabbu \\ Department of BME \\ Osmania University, Hyderabad, \\ Telangana, India - 500007
}

\author{
Malini Mudigonda \\ Department of BME \\ Osmania University, Hyderabad, \\ Telangana, India - 500007
}

\begin{abstract}
Advancements in the field of computational systems have enabled decoding of biological quantities and to recognize physiological conditions. One such prospective application for the next generation of portable electroencephalogram (EEG) signal processing systems is to prevent hazards in attention-demanding activities. This work demonstrates a methodology for assessing alertness level based on a single EEG channel (PzOz), allowing the reduction of the required hardware and the computational time of the algorithms. A new spectral power-based index is proposed and computed through the normalized Haar discrete wavelet packet transform (WPT). The Haar WPT precisely resolves the brain rhythms into packets whilst demanding a relatively low computational cost. The effectiveness of the proposed index in drowsiness detection is evaluated by evidencing the significant changes in the alert-drowsy transitions of 40 subjects of a public database. It can be clearly seen from the results that the proposed index is distinctive enough to distinguish active and drowsy states $(0.25)$ based on frequency spectrum states contained in the EEG signal.
\end{abstract}

\section{Keywords}

Electroencephalogram, Wavelet Packet Analysis, Haar Wavelet, Spectral Power based Index

\section{INTRODUCTION}

An ever-rising deadly toll due to vehicle based accidents makes it one of the major concerns in this traffic prevalent era. One of the major root cause of such accidents is due to fatigue or drowsiness whilst driving. Drowsy state can be characterized by the decrease of the subject's information processing speed and memory capacities occurring when one is about to fall asleep. Such fatigue may originate from various attributes such as effect of alcohol or other intoxicants, even lack of sleep or prolonged driving. Examples of professionals under high risk include, but not restrict to, flight pilots, air traffic controllers and lorry and regular drivers.

In a recent AAA survey of highway vehicles in 2016, for example, 43 percent of U.S. drivers admitted to falling asleep or nodding off while driving at least once in their lifetime [1, 2]. Every 1 in
25 adult drivers (aged 18 years or older) have reported to be fallen asleep while driving in the a span of 30 days [3]. The National Highway Traffic Safety Administration estimates that close to 72,000 crashes, 44,000 injuries, and 800 deaths arised due to drowsy driving in 2013 alone. These figures are underestimated and in reality maybe up to 6,000 fatal crashes are caused by drowsy drivers each year [3]. Not just driving on roads but pilots (23\%) and almost one-fourth of train operators $(26 \%)$ admit that sleepiness has had an impact on their job performance once a week at least, compared to one in six non-transportation workers (17\%) [4, 5]. India's National Crime Records Bureau does not record accidents caused by tired drivers but senior police officers say that a number of accidents are caused by people dropping off behind the wheel. Many drivers do not realize that they are sleepy. Sometimes they cause major accidents within seconds. This has led to a necessity for various methodologies to overcome or compensate for this state of drowsiness. Technologically driven solutions have been proven to be robust and accurate enough to detect fatigue conditions while driving scenarios.

Increasing traffic density, road complexity and implementation of in-vehicle technologies (IVTs), such as navigation system, cellular phone, in-vehicle entertainment systems, etc., the driving task has become a more complex and interactive activity rather than a simple act of mobility. In such situations, the driver's attention is increasingly diverted from the primary task of driving and hazard detection. As a measure, slowly high end technology, which enables cars to be fully automated e.g. adaptive cruise control or ACC system enables automatic car driving without driver's input in the highway are being implemented. It is of utmost importance to ensure that the driver is in the loop on higher levels of automation when required so that he is able to react properly to a potential critical situation. Therefore it is a promising path to develop systems that can automatically detect when a driver is moving into a fatigue phase and accordingly alert impromptu.

Previously lot of research has been performed on ways to detect drowsiness and integrate it with technological solutions. Some of the most common ways to do this are assessing the driver's current state, especially relating to the eye and eyelid movements physiological state changes. Or to gauge driver performance, with a focus on the vehicle's behavior including lateral position 
and headway and in some cases a combination of the driver's current state and driver performance. They may consider a wide range of inputs, as facial movements (Aoi, Kamijo, Yoshida, 2011 [6]; Gu, Ji, Zhu, 2002 [7]), eyes closures (Tsuchida, Bhuiyan, Oguri, 2009 [9]; Wierwille, Wreggit, Kirn, et al., 1994 [10]) and physiological signals as electromyogram (EMG) (Aoi et al., 2011 [6]), electrocardiogram (ECG) (Zhang and Liu, 2012 [11]), electrooculogram (EOG) (Chieh, Mustafa, Hussain, Hendi, Majlis, 2005 [15]) and electroencephalogram (EEG) (Eoh et al., 2005 [19] and Subasi, $2005[16]$ ). However, it is argued that EEG signals are the most reliable indicators of drowsiness due to direct correlation and thus promising techniques can be developed for detection.

It is well established in literature the existence of special frequency ranges of EEG signals (called rhythms) (Bear, Connors, Paradiso, 2007 [17]) when measured on the scalp, those rhythms are faded expressions of the characteristic synchrony between populations of neurons that appear more or less intensely in each phase of the circadian cycle. Through analysis of short time intervals of the raw signal, labeled as epochs, it is possible to recognize the subjects ' brain activities, described by the different rhythms. For example, the delta rhythm increases during high sleep and also can indicate the need for sleep (Subasi, $2005[16]$ ). The theta activity gradually increases whilst the alpha rhythm decreases from the wakefulness to resting conditions (Kiymik, Akin, Subasi, 2004 [18]). High and low activities in beta band are related to the alert and drowsy states, respectively (Eoh et al., 2005 [19]). Eoh et al. (2005) [19] employed these knowledge, proposing and analyzing indices based only on the power spectrum of brain activities. They have included the theta, alpha and beta rhythms in their studies, analyzing these rhythms" behavior and the relationship between them. Respectively, in their studies, 8 and 30 EEG channels were used to perform the experiments in driving simulation tasks.

Albeit not stating explicitly, as Eoh et al. (2005) [19] averaged all channels to compute rhythm energies and employed Analysis of Variance (ANOVA) to choose the electrodes containing significant variations, their results suggest drowsiness is detectable with fewer EEG inputs. Furthermore, the demand for methodologies based on fewer channels (Yu et al., 2013 [21]) has increased in the present decade because of the blooming of portable devices. Thanks to the increasing power and popularity of portable electronic systems, those like the one proposed in Lin et al. (2010) [22] became an attractive option to process EEG and other physiological signals. According to Zhu, Li, and Wen (2014) [23], multi-channel equipment limits the subjects" movements and are harder to use than single-channel devices. In this context, contrasting to the literature on the sleep stages scoring problem (Ebrahimi, et al., 2008 [24]; Fraiwan, et al., 2012 [25]; Zhu et al., 2014 [23]), few studies have regarded this relevant requirement for drowsiness detection systems and methods (Lin et al., 2010 [22]; da Silveira, et al., 2015 [26]).

Therefore, this work is an effort to develop an automated drowsiness detection system using Wavelet analysis on a single channel Electroencephalogram (EEG) signal. Additionally, a new index has been proposed that can fasten the computation and decrease the delay as a continuation to previous work on drowsiness detection [27, [12 [8, 20]. This paper shall address three major aspects: (1.) acquisition of the EEG signals from the subjects during active and drowsy conditions. (2.) differentiating the intervals in drowsy state from active state from the EEG signals. (3.) proposal and discus- sion on a new index that reducing the computational time and cost whilst giving accurate results.

\section{BACKGROUND AND METHODOLOGY}

\subsection{Data handling}

The experimental data used in this study were obtained from the public Sleep EDF database [expanded] part of the Physionet Bank [28]. One of the reason towards using such information from a public database is to enable the possibility of testing, verifying and extending the proposed methodology by any research group interested in this subject. This database provides recordings from 40 healthy subjects. This collection of polysomnograms (PSGs) with accompanying hypnograms (expert annotations of sleep stages) comes from two studies.

The *PSG.edf files are whole-night polysmnographic sleep recordings containing EEG (from Fpz-Cz and Pz-Oz electrode locations), EOG (horizontal), submental chin EMG, and an event marker; the SC*PSG.edf files often also contain oro-nasal respiration and rectal body temperature. Each EDF and EDF+ file has a header specifying the patient (in these files anonymized to only gender and age), details of the recording (in particular the recorded time period), and characteristics of the signals including their amplitude calibration.

The recording of all available subjects, who stated not having used any sleep-related medication while participating in the experiments, were used. These available recordings refer to male and female subjects with ages between 20 and 34 years. They contain for each subject's recording two EEG channels, Fpz- $\mathrm{Cz}$ and $\mathrm{Pz}-\mathrm{Oz}$, sampled at $100 \mathrm{~Hz}$ besides EOG and EMG signals. The EOG and EEG signals were each sampled at $100 \mathrm{~Hz}$. In the current study, the $\mathrm{Pz}-\mathrm{Oz}$ channel is adopted, because this channel is to be more accurate for scoring sleep stages. Furthermore, [13] found that $\mathrm{Oz}$ location can provide discriminating power and have high correlation with the drowsiness state. [14] have found similar results, but considering EEGs from the parietal region. Annotations part of the hypnograms provided for every 30 seconds are extremely useful, as they are reliable delimiters to point out when the subjects are considered alert, drowsy (S1 stage) or sleeping.

\subsection{Hypnogram studies}

Hypnogram are as important as data files because they are encoded with annotations i.e. sleep stages at different time intervals. The *Hypnogram.edf files contain annotations of the sleep patterns that correspond to the PSGs. These patterns (hypnograms) consist of sleep stages W, R, 1, 2, 3, 4, M (Movement time). All hypnograms were manually scored by well-trained technicians (identified by the eighth letter of the hypnogram filename).

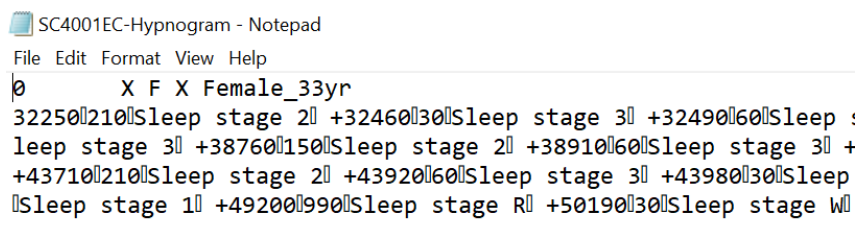

Fig. 1. Hypnogram in notepad (clearly the data is encoded with the age, gender and timestamps for sleep cycles 


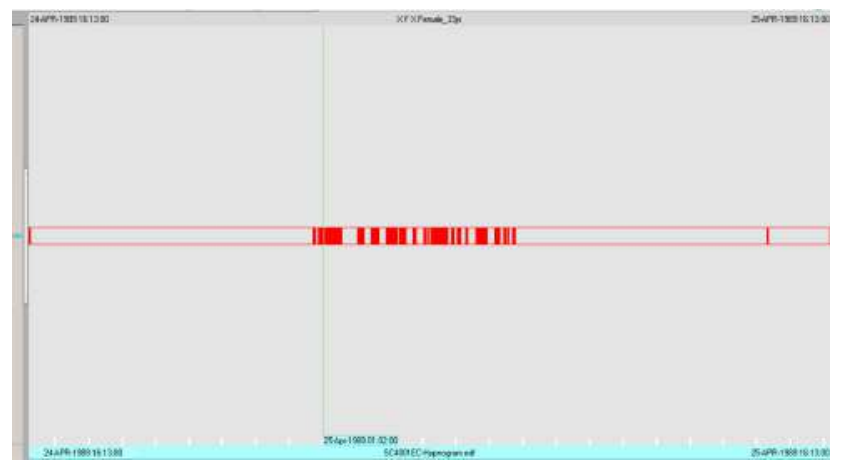

Fig. 2. Hypnogram in Polyman software shows the timestamps of sleep modes.

\subsection{Wavelet Transformations}

Non-stationary data with different frequency properties, as the EEG signals, can be successfully analyzed through the wavelet transform application. The wavelet transform also provides a multi-resolution analysis in both time and scaling domains, differently from the Fourier transform, though frequency and scale are related. Several wavelet transforms, with different basis and decomposition algorithms, have already been explored in the sleep research field. The discrete wavelet transform (DWT) was used as a tool to recognize the alertness level in whilst the wavelet packet transform (WPT) algorithm and the continuous wavelet transform were used to classify sleep stages respectively. In the current study, the Haar WPT with normalized filters is adopted.

$$
\psi(t)= \begin{cases}1 & 0 \leq t<1 / 2 \\ -1 & 1 / 2 \leq t<1 \\ 0 & \text { otherwise }\end{cases}
$$

The Haar wavelet's mother wavelet function $\psi(\mathrm{t})$ can be described as equation 1 Haar basis being the simplest wavelet, avoiding any special boundary treatment makes it possible to apply the packet transform algorithm over each EEG epoch without insertion of artificial values or loss of information. Figure 3 presents the structure of the packet transform, showing the many packets obtained by level, each one containing different sets of wavelet coefficients, generated by the decomposition of the scaling and wavelet packets previously computed.

Aiming applications in real time, the current study uses epochs of 30 s length. Once the considered epochs have $30 \mathrm{~s}$ length and the provided Physionet hypnograms refer to 30 -s ones. Moreover, once the sample rate of the analyzed EEG signal is $100 \mathrm{~Hz}$, each epoch has 30000 samples. The application of the WPT algorithm allows to break down the whole data (30-s epoch) in specific frequency ranges between 0 and the half of the signals' sample rate, i.e. 50 $\mathrm{Hz}$. Therefore, wavelet packet coefficients related to frequency ranges of the delta $(0.5-4 \mathrm{~Hz})$, alpha $(8-13 \mathrm{~Hz})$, beta $(13-30$ $\mathrm{Hz})$ and low-gamma $(30-50 \mathrm{~Hz})$ rhythms are selected. The delta rhythm is the slowest wave activity, gamma is the fastest one. As already mentioned, the former is associated to the need for sleep while the later, to the working memory and attention. Given these associations with the cognitive state of humans, it is expected that both rhythms will be of interest to identify the trend to the drowsy state.

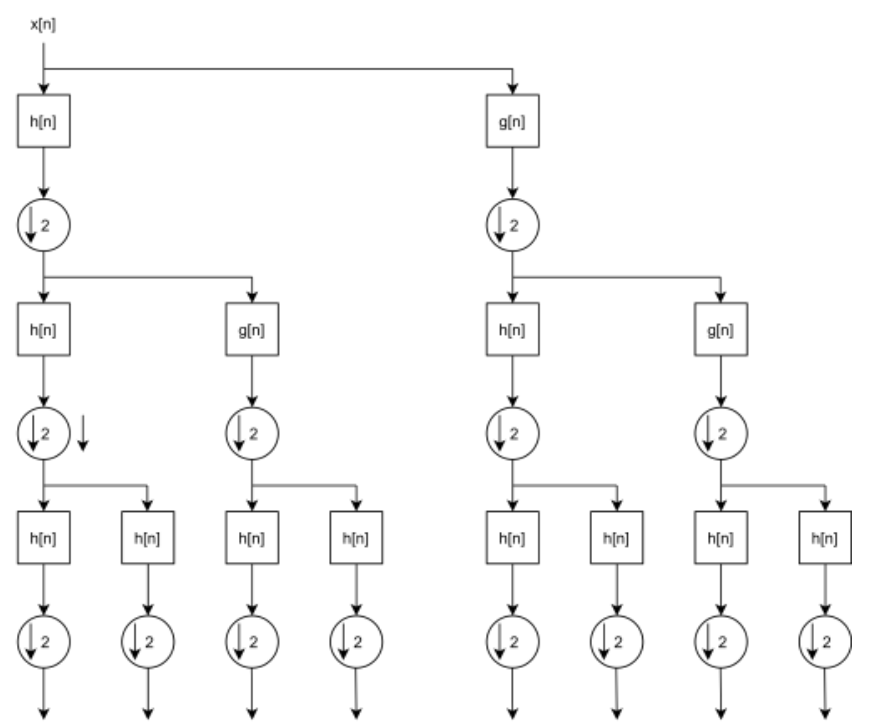

Fig. 3. Wavelet packet decomposition

Preliminary experiments identified a complementary and sensitive behavior of these rhythms to the alert-drowsy transition as pointed out in the following subsections. Furthermore, here, alpha and beta rhythms are also adopted since their effectiveness was already demonstrated in several studies related to the sleep and drowsiness analysis. After the normalized Haar WPT application, wavelet packet set of coefficients (WPsc) are selected.

The WPsc corresponding to the delta rhythm are contained in packets labeled as $C_{5,1}, C_{6,1}, C_{6,4}$ and $C_{8,3}$ according to Figure 4 Alpha and beta information are inside, respectively, $C_{4,3}, C_{4,11}$, $C_{7,21}, C_{7,32}, C_{8,41}$ and $C_{9,132}$, and $C_{3,3}, C_{4,5}, C_{4,8}, C_{5,9}, C_{5,18}$, $C_{6,17}, C_{8,67}, C_{8,152}$ and $C_{9,306}$. Low-gamma data are within the blocks $C_{2,3}, C_{3,5}, C_{6,39}$ and $C_{7,77}$. The assumed notation refers to the WPsc as $C_{i, j}$ with i 0 being the binary tree level and $\mathrm{j} \in$ $[0,2 \mathrm{i}-1]$ indicating the $\mathrm{j}$-th set at level $\mathrm{i}$. Since the employed transform is decimated, a set of coefficients at a level $i$ has half of the number of coefficients with respect to the set at the level i- 1 . The covered frequency ranges for the rhythms of interest are summarized in Table. 1.

Table. 1. Frequency ranges of different brain waves

\begin{tabular}{|l|l|c|}
\hline Brain Rhythms & $\begin{array}{l}\text { Actual fre- } \\
\text { quency range }\end{array}$ & Covered frequency range \\
\hline Delta & $0.5-4$ & $0.5859375-3.90625$ \\
\hline Theta & $4-8$ & $4.023475-7.892212375$ \\
\hline Alpha & $8-13$ & $8.0078125-12.98828125$ \\
\hline Beta & $13-30$ & $13.0859375-29.98046875$ \\
\hline Low-Gamma & $30-50$ & $30.078125-50$ \\
\hline
\end{tabular}

Note that, according to Table. 1, the length of the analyzed epochs and the sample rate of the EEG signals are good enough to well represent the bands of the regarded brain rhythms. Figure 4 illustrates the selected WPsc for delta, alpha, beta and low-gamma rhythms. Note that $C_{0,0}$, the input set (epoch), contains $\mathrm{L}=30,000$ 


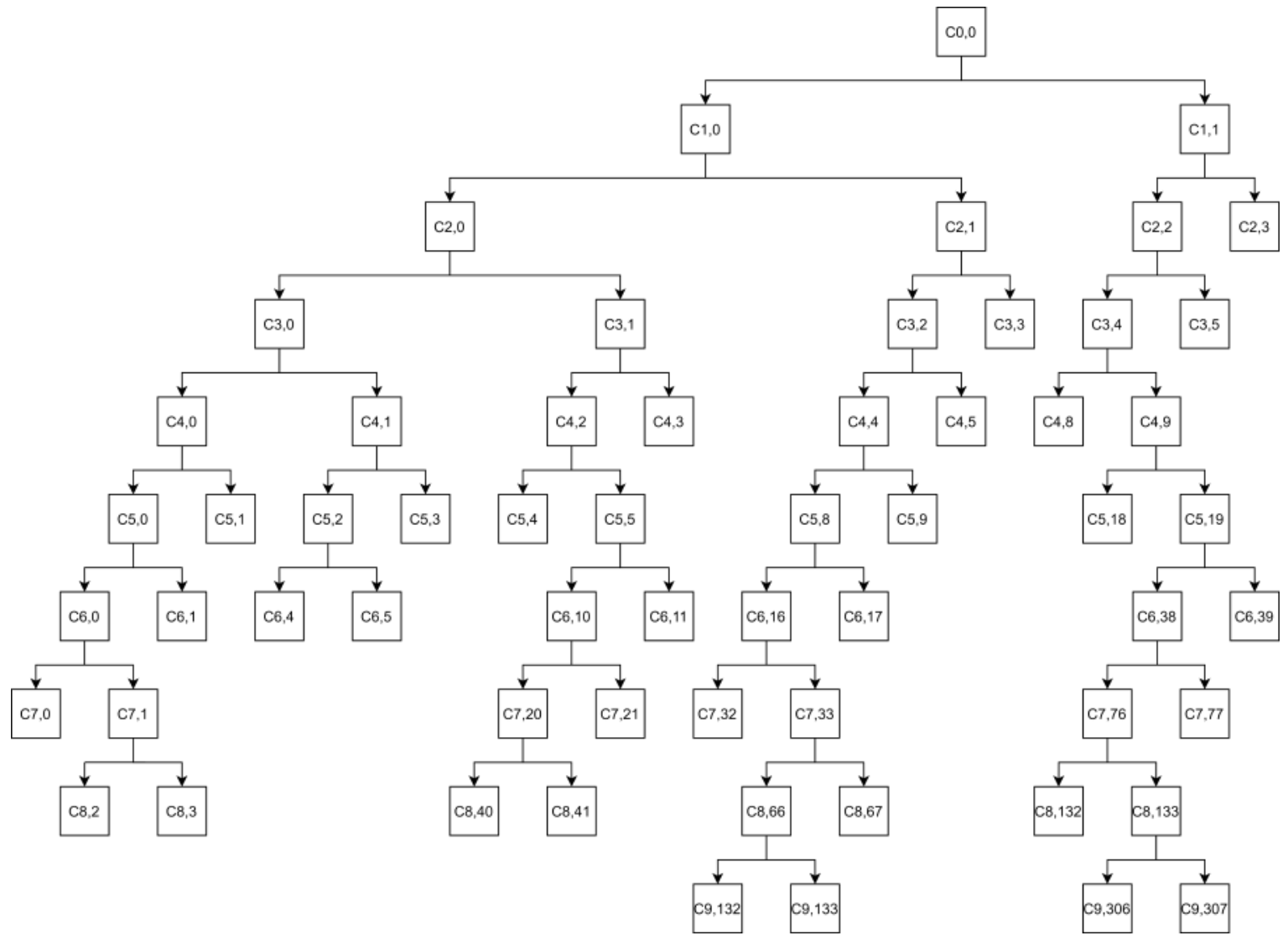

Fig. 4. Wavelet packet decomposition for this study

discrete points and is sampled at $100 \mathrm{~Hz}$. By considering these parameters, it is possible to analyze the information within each WPsc with reliable frequency representation and few decomposition levels. It is important to remind that altering the sample rate and/or the quantity of points in $\mathrm{C} 0,0$, the WPT structure will change as well.

Another important aspect to be highlighted is the relevance of choosing the normalized Haar filters. Once all indices are based on energy computations, they would be distorted by non-normalized transforms. Given the WPsc related to the delta, alpha, beta and low gamma rhythms, it is possible to calculate their powers in such epoch. Due to the normality and orthogonality of the Haar basis, it can be made by summing up the powers of all sets of coefficients related to the whole frequency range of interest. Thus, the delta $(\delta)$, alpha $(\alpha)$, beta $(\beta)$ and low-gamma $(\gamma)$ powers are given, respectively, by

$$
\begin{gathered}
\delta=P\left(C_{5,1}\right)+P\left(C_{6,1}\right)+P\left(C_{6,4}\right)+P\left(C_{8,3}\right) \\
\alpha=P\left(C_{4,3}\right)+P\left(C_{4,11}\right)+P\left(C_{7,21}\right) \\
+P\left(C_{7,32}\right)+P\left(C_{8,41}\right)+P\left(C_{9,132}\right) \\
\gamma=P\left(C_{2,3}\right)+P\left(C_{3,5}\right)+P\left(C_{6,39}\right)+P\left(C_{7,77}\right)
\end{gathered}
$$

$$
\begin{array}{r}
\beta=P\left(C_{3,3}\right)+P\left(C_{4,5}\right)+P\left(C_{4,8}\right)+P\left(C_{5,9}\right)+P\left(C_{5,18}\right) \\
+P\left(C_{6,17}\right)+P\left(C_{8,67}\right)+P\left(C_{8,152}\right)+P\left(C_{9,306}\right)
\end{array}
$$

Thus the power of the signal is given by

$$
P\left(C_{i, j}\right)=\sum_{l=0}^{k-1}\left(C_{i, j}(l)\right)^{2}
$$

The value of $C_{i, j}$ is the $l^{t h}$ coefficient of the set $C_{i, j}$ and $\mathrm{K}=\mathrm{L} / 2^{i}$ is its cardinality.

\section{RESULTS}

Real data set of 40 subjects have been used to detect 'Drowsy' and 'Active' states. The EEG data of Pz-Oz channel has been extracted and then segmented as per the frequency range of the signal and wavelet packets using Haar transformation. Delta, Alpha, Beta, Gamma powers of the subjects have been used to find the proposed index value at different sleep stages. Using the index value which is obtained from the data sets, drowsy and active states can be differentiated. The following graphs and statistics are the output results of the spectral analysis of EEG data of reference subject after converting from EDF to ASCII. 
Subsequently various spectral powers within the data have been computed which shall help us evaluate the index to differentiate drowsy and active states. Since the power of the signal is given by equation (6), the relative power of a specific signal in spectrum is given by

Relative power $=($ Power of the specific signal $) /($ Total power $)$

Using this principle, the powers of various states like alpha, delta, gamma and beta have been estimated and the relative power is the corresponding state power/total power of the signal. These plots can be obtained based on equations (2), (3), (4), (5) respectively.

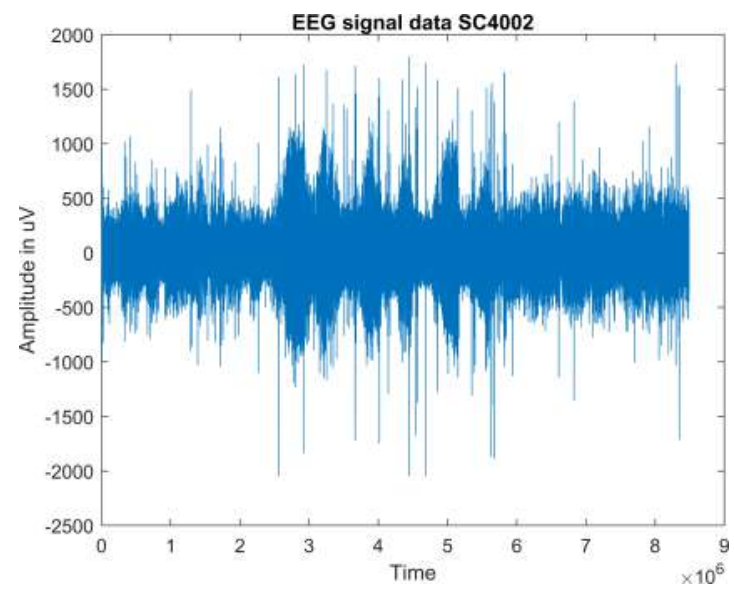

Fig. 5. EEG data of SC4002

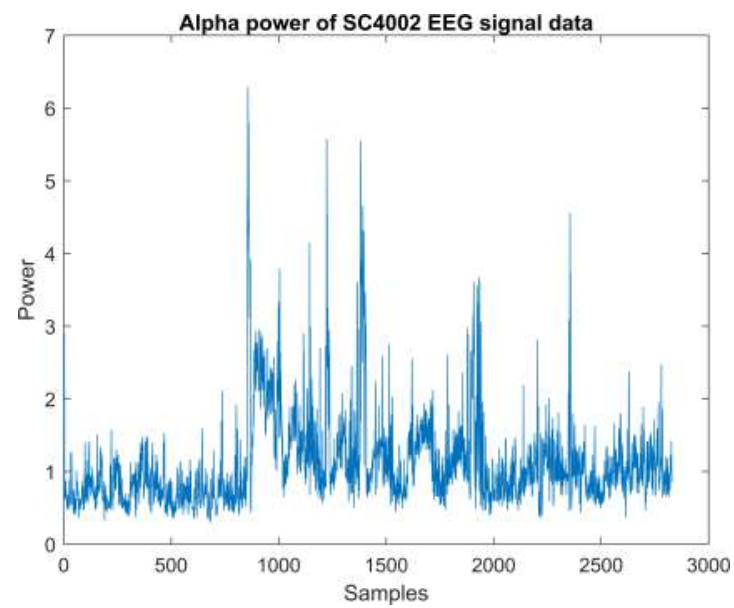

Fig. 6. Alpha power for the EEG data of SC4002

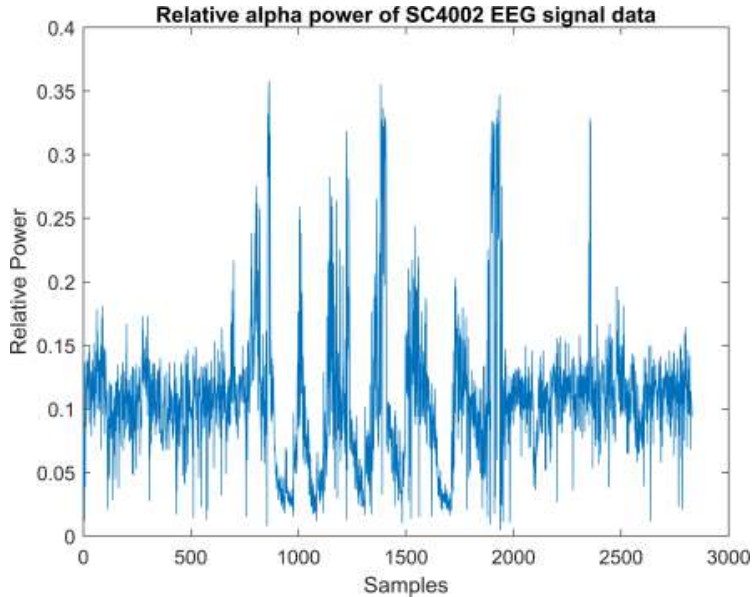

Fig. 7. Relative Alpha power for the EEG data of SC4002

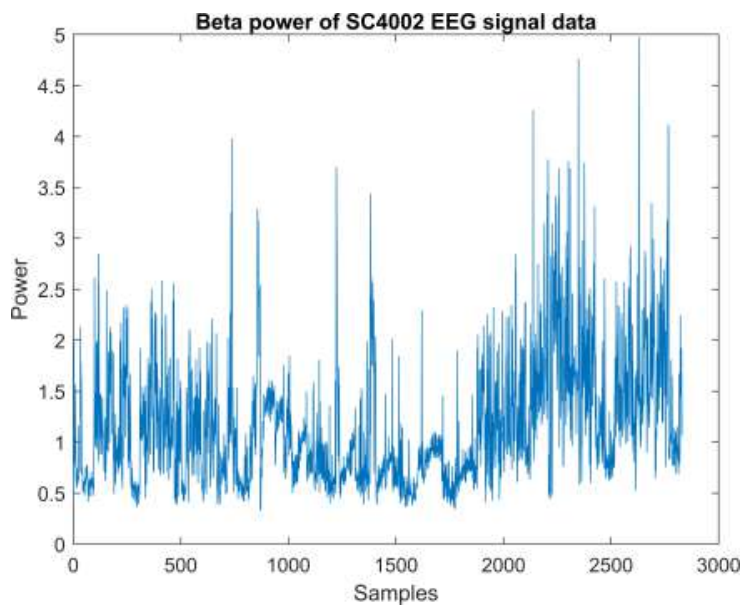

Fig. 8. Beta power for the EEG data of SC4002

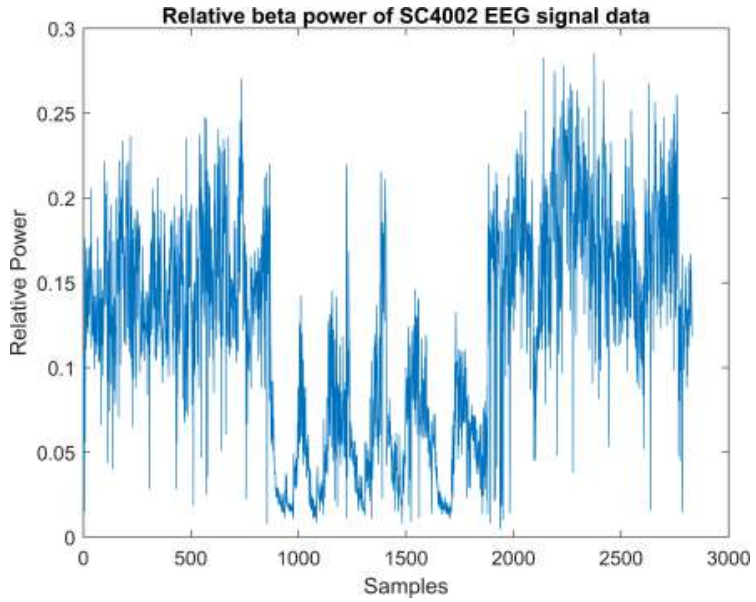

Fig. 9. Relative Beta power for the EEG data of SC4002 


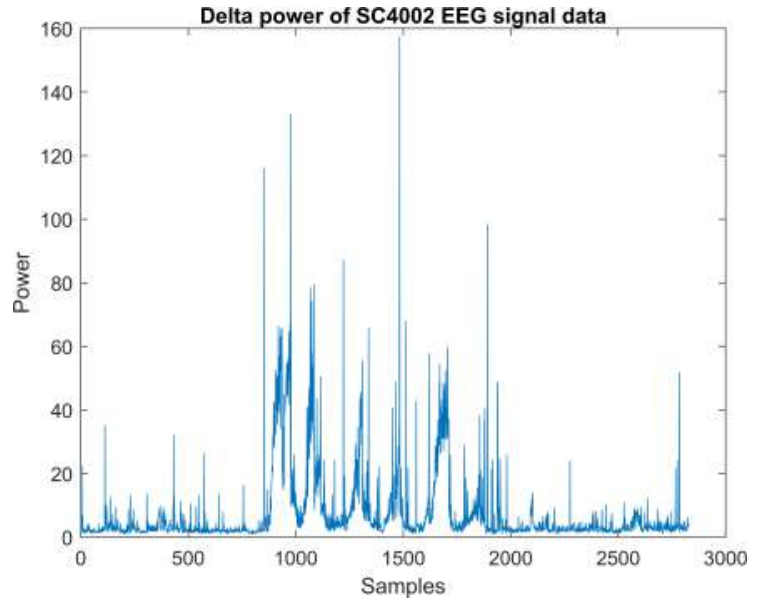

Fig. 10. Delta power for the EEG data of SC4002

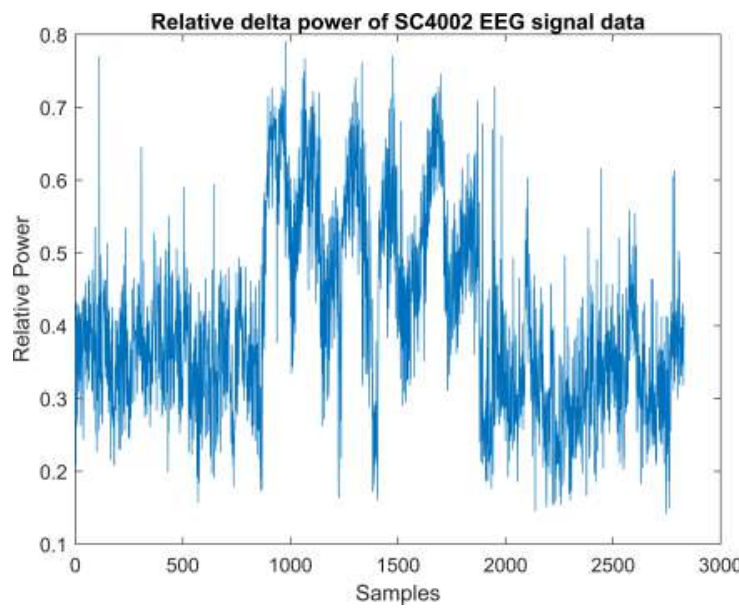

Fig. 11. Relative Delta power for the EEG data of SC4002

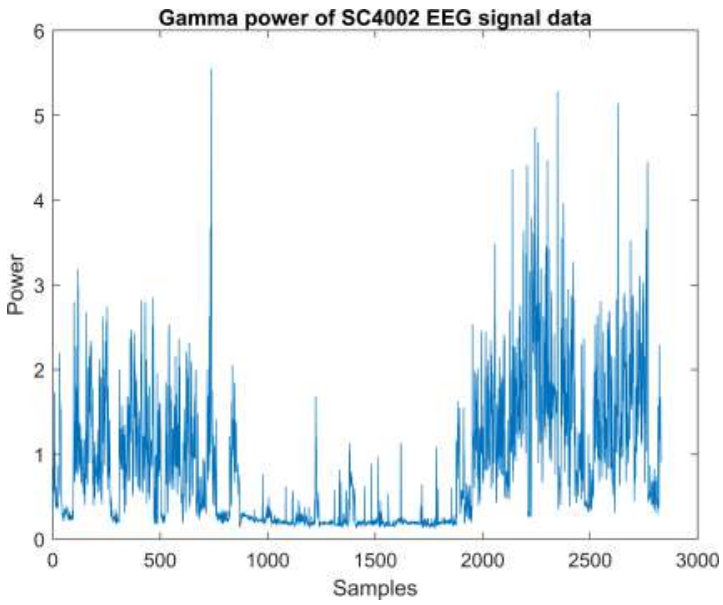

Fig. 12. Gamma power for the EEG data of SC4002

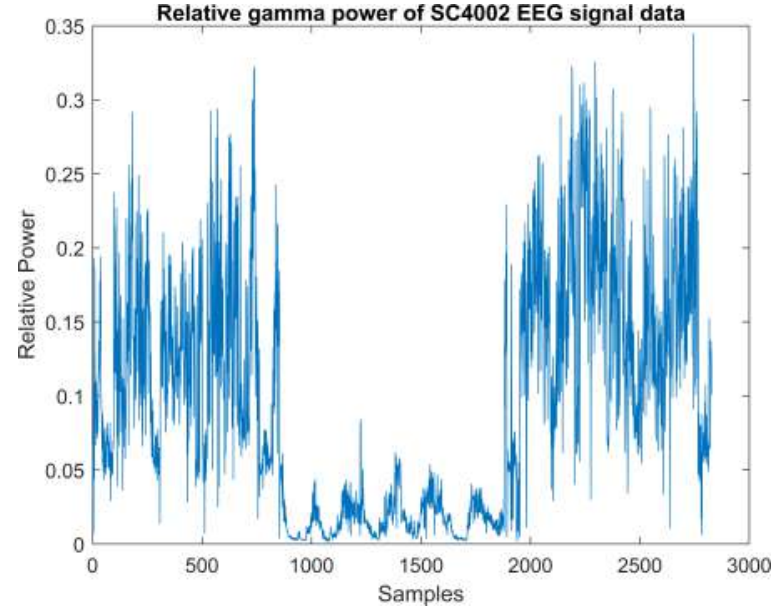

Fig. 13. Relative Gamma power for the EEG data of SC4002

From this point, a statistical study has been performed to find the average powers of the different sleep stages (for the Pz-Oz channel) are calculated using the EEG data and the sleep annotations. Using the sleep annotations data is separated, that is samples of different sleep stages are separated and then the averages of relative powers have been calculated. These values for the forty reference subjects are studied and the trends in the average values of the power in drowsy and active modes have been shown.

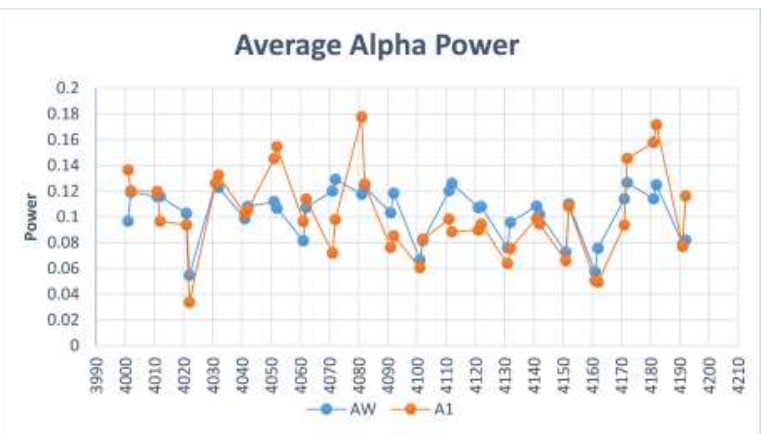

Fig. 14. Average Alpha power for all the subjects in active and drowsy states

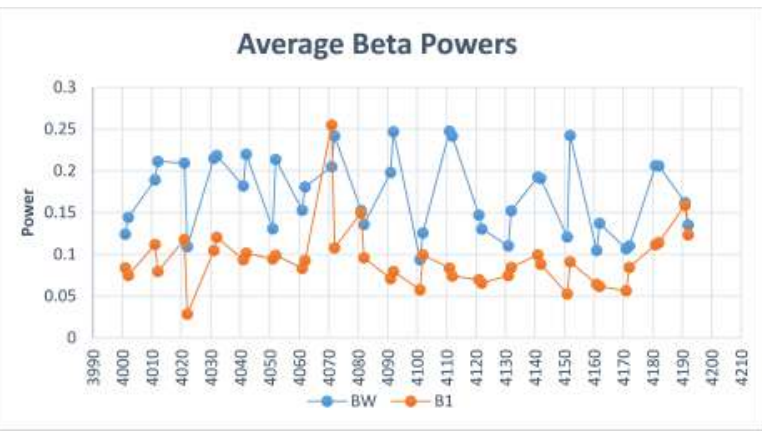

Fig. 15. Average Beta power for all the subjects in active and drowsy states 


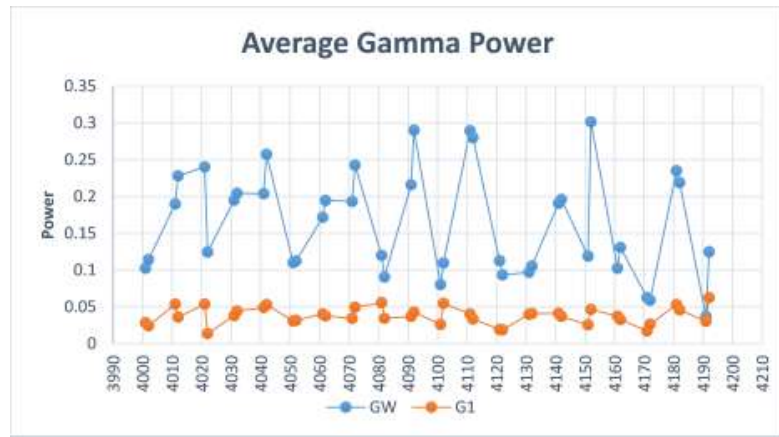

Fig. 16. Average Gamma power for all the subjects in active and drowsy states

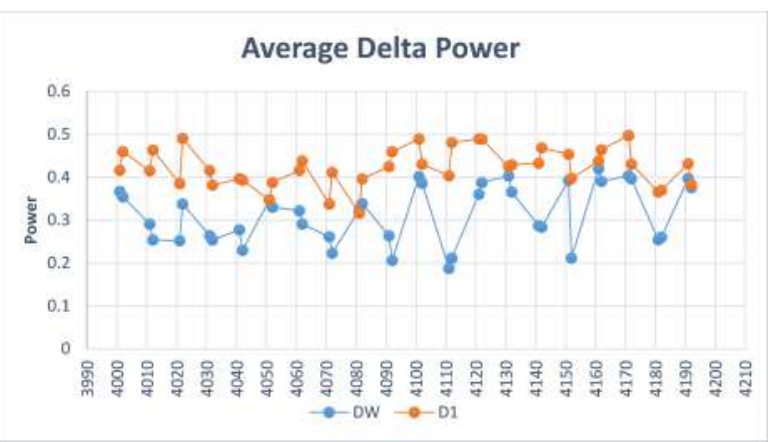

Fig. 17. Average Delta power for all the subjects in active and drowsy states

On the basis of these values of the independent spectrum powers, the average index value is estimated along with the analysis of index value from the signal data and the corresponding graph and characteristics have been obtained.

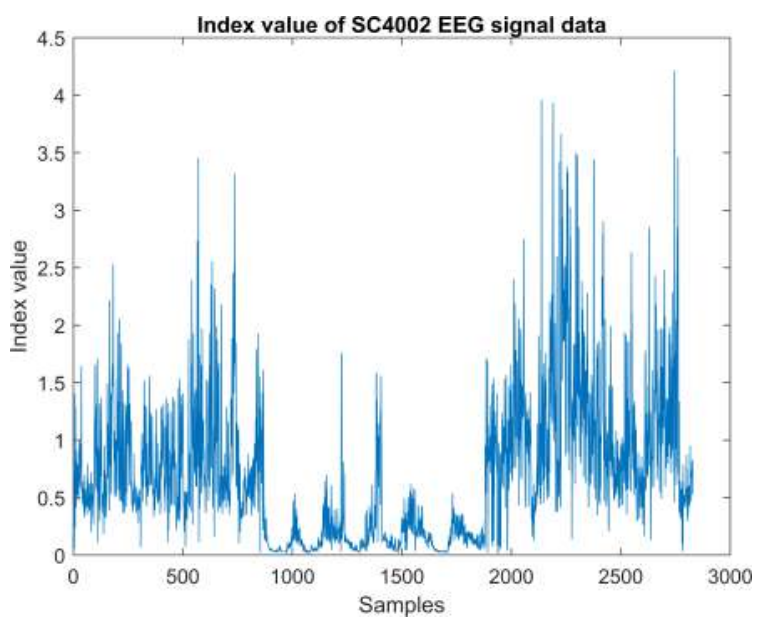

Fig. 18. Index value for the EEG data of SC4001 low index value( $\mathrm{I}<0.25)$ represents drowsy state

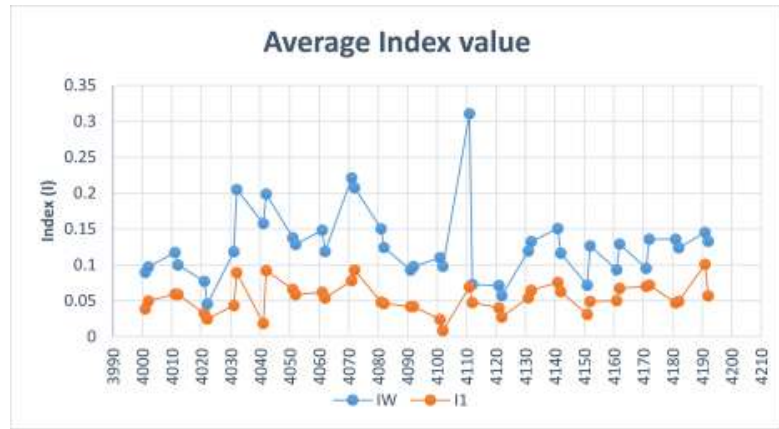

Fig. 19. Average Index value for all the subjects in active and drowsy states

\section{CONCLUSION}

Observing average powers of different wavelets in different stages states it can be deduced that:

- Changes in the alpha power are very small during the transition from awake to drowsy.

- Power decreases from wake state to drowsy state during beta stage.

- Similarly gamma power also decreases from wake state to drowsy state.

- Delta power increases from wake state to drowsy state.

- Delta is maximum during the sleep stage four.

Based on the analysis, it is clearly observed that the power of gamma and beta decreases whereas power of delta increases.

Therefore the new ratio index is introduced as

$$
\operatorname{Index}(I)=(\gamma+\beta) /(\delta),
$$

where $\delta, \alpha, \beta$ and $\gamma$ are defined, respectively, in the equations (2) (5). It can clearly be seen from the index values for EEG data and the average index values for different subjects, that the Index (I) value decreases from active state to drowsy state. It can be characterized that for this case, the subject is drowsy for values of index less than 0.25 .

\section{REFERENCES}

[1] Reduce Fatigue-Related Accidents based on 2002 survery, National transportation safety board. https://www.ntsb.gov/safety/mwl/Pages/mwl1-2016.aspx

[2] Tefft BC, AAA Foundation for Traffic Safety. Prevalence of Motor Vehicle Crashes Involving Drowsy Drivers, United States, 2009 - 2013[457 KB].Washington, DC: AAA Foundation for Traffic Safety; 2014. October 19, 2015.

[3] National Highway Traffic Safety Administration. Research on Drowsy Driving. Accessed October 20, 2015, Center for Disease Control and Prevention

[4] Masten SV, Stutts JC, Martell CA. Predicting daytime and nighttime drowsy driving crashes based on crash characteristic models. 50th Annual Proceedings, Association for the Ad- 
vancement of Automotive Medicine; October 2006; Chicago, IL.

[5] Institute of Medicine and sleep foundation. Sleep Disorders and Sleep Deprivation: An Unmet Public Health Problem. Washington, DC: The National Academies Press; 2006.

[6] M. Aoi, M. Kamijo and H. Yoshida, "Relationship between Facial Expression and Facial Electromyogram (f-EMG) Analysis in the Expression of Drowsiness," 2011 International Conference on Biometrics and Kansei Engineering, Takamatsu, Kagawa, 2011, pp. 65-70. doi: 10.1109/ICBAKE.2011.26

[7] Haisong Gu, Qiang Ji and Zhiwei Zhu, ”Active facial tracking for fatigue detection," Sixth IEEE Workshop on Applications of Computer Vision, 2002. (WACV 2002). Proceedings., Orlando, FL, USA, 2002, pp. 137-142. doi: 10.1109/ACV.2002.1182171

[8] Vamsi R., Suman D., CH N., Malini M. (2017) Discrete Wavelet Transform based statistical features for the Drowsiness detection from EEG. In: Goh J., Lim C., Leo H. (eds) The 16th International Conference on Biomedical Engineering. IFMBE Proceedings, vol 61. Springer, Singapore

[9] A. Tsuchida, M. S. Bhuiyan and K. Oguri, "Estimation of drowsiness level based on eyelid closure and heart rate variability," 2009 Annual International Conference of the IEEE Engineering in Medicine and Biology Society, Minneapolis, MN, 2009, pp. 2543-2546.

[10] Wierwille, W W; Wreggit, S S; Kirn, C L; Ellsworth, L A Fairbanks, R J, "Research on vehicle-based driver status/performance monitoring; development, validation, and refinement of algorithms for detection of driver drowsiness, December 23, 1994. National Highway Traffic Safety Administration.

[11] A. Zhang and F. Liu, "Drowsiness detection based on wavelet analysis of ECG and pulse signals," 2012 5th International Conference on BioMedical Engineering and Informatics, Chongqing, 2012, pp. 491-495. doi: 10.1109/BMEI.2012.6513058

[12] D. Suman, M. Malini, M. Venkateswara Rao, "Design and development of a comprehensive driver drowsiness detection and alerting system", International Journal of Advances in Science and Technology (IJAST), pp. 57-60, 2014.

[13] Pal, N.R., Chuang, CY., Ko, LW. et al. EEG-Based Subjectand Session-independent Drowsiness Detection: An Unsupervised Approach. EURASIP J. Adv. Signal Process. 2008, 519480 (2008). https://doi.org/10.1155/2008/519480

[14] Maglione A, Borghini G, Aricò P, Borgia F, Graziani I, Colosimo A, Kong W, Vecchiato G, Babiloni F. Evaluation of the workload and drowsiness during car driving by using high resolution EEG activity and neurophysiologic indices. Annu Int Conf IEEE Eng Med Biol Soc. 2014;2014:6238-41. doi: 10.1109/EMBC.2014.6945054. PMID: 25571422.

[15] Thum Chia Chieh, M. M. Mustafa, A. Hussain, S. F. Hendi and B. Y. Majlis, "Development of vehicle driver drowsiness detection system using electrooculogram (EOG)," 2005 1st International Conference on Computers, Communications, \& Signal Processing with Special Track on Biomedical Engineering, Kuala Lumpur, Malaysia, 2005, pp. 165-168.

[16] Subasi, A., "Automatic recognition of alertness level from EEG by using neural network and wavelet coefficients," Experts Systems with Applications, 2005, 28(4), 701-711.
[17] Bear, M. F., Connors, B. W., \& Paradiso, M. A. (2007). Neuroscience: Exploring the brain (3rd ed.). Philadelphia, PA, US: Lippincott Williams \& Wilkins Publishers.

[18] Kiymik, M. K., Akin, M., \& Subasi, A. (2004). Automatic recognition of alertness level by using wavelet transform and artificial network. Journal of Neuroscience Methods, 139(2), 231-240.

[19] J. Eoh, Hong \& K. Chung, Min \& Kim, Seong-Han. (2005). Electroencephalographic study of drowsiness in simulated driving with sleep deprivation. International Journal of Industrial Ergonomics. 35. 307-320. 10.1016/j.ergon.2004.09.006.

[20] Dabbu, S., Malini, M., Reddy, B.R. and Vyza, Y.S.R., 2017. ANN based Joint Time and frequency analysis of EEG for detection of driver drowsiness. Defence Life Science Journal, 2(4), pp.406-415.

[21] Yu, S., Li, P., Lin, H., Rohani, E., Choi, G., Shao, B., \& Wang, Q. (2013).Support vector machine based detection of drowsiness using minimum EEG features. In Proceedings of 2013 international conference on social computing (SOCIALCOM) (pp. 827-835). doi:10.1109/SocialCom.2013.124.

[22] Lin, C.-T., Chang, C.-J., Lin, B.-S., Hung, S.-H., Chao, C.F., \& Wang, I.-J. (2010). A real-time wireless brain-computer interface system for drowsiness detection. IEEE Transactions on Biomedical Circuits and Systems, 4(4), 214-222.

[23] Zhu, G., Li, Y., \& Wen, P. (2014). Analysis and classification of sleep stages based on difference visibility graphs from single channel EEG signal. IEEE Journal of Biomedical and Health Informatics, 18(99), 1-9. doi:10.1109/JBHI.2014.2303991.

[24] Ebrahimi, F., Mikaeili, M., Estrada, E., \& Nazaren, H. (2008). Automated sleep stage classification based on EEG signals by using neural networks and wavelet packet. In Proceedings of the 30th annual international IEEE engineering in medicine and biology society, EMBS conference (pp. 1151-1154).

[25] Fraiwan, L., Lweesy, K., Khasawneh, N., Wenz, H., \& Dickhaus, H. (2012). Automated sleep stage identification system based on time-frequency analysis of a single eeg channel and random forest classifier. Computer Methods and Programs in Biomedicine, 108(1),10-19.

[26] da Silveira, T., Kozakevicius, A. J., \& Rodrigues, C. R. (2015). Drowsiness detection for single channel EEG by DWT best $\mathrm{m}$-term approximation. Research on Biomedical Engineering, 31, 107-115.

[27] D. Suman, M. Malini and S. Anchuri, "EOG based vigilance monitoring system," 2015 Annual IEEE India Conference (INDICON), New Delhi, 2015, pp. 1-6. doi: 10.1109/INDICON.2015.7443210

[28] Physionet (2013). The sleep-EDF-X database. http://www.physionet.org/physiobank/ database/sleep-edfx. Online; Accessed online. 